\title{
A minicomputer-based learning analysis system for optimizing PSI instructional materials for the visually handicapped
}

\author{
THOMAS B. PERERA \\ Montclair State College, Upper Montclair, New Jersey 07043 \\ and \\ EDWARD S. COBB \\ Bronx Community College, Bronx, New York 10453
}

\begin{abstract}
Visually handicapped students learn tape-recorded materials in PSI format while a minicomputer interfaced to the student-operated, tape playback mechanisms collects data on independent study activities. The minicomputer hardware and software system is described and typical learning data are discussed.
\end{abstract}

The "Learning Through Listening" project, funded by the Bureau of Special Continuing Education of the New York State education department was established to develop new and more efficient techniques for teaching visually handicapped students using tape-recorded instructional materials. A major goal of the project is the development of optimized, selectable-channel tape recordings for independent study as an alternative to the literal transcriptions of textbooks. The format of the new tape recordings, one largely developed from procedures used in the Personalized System of Instruction (PSI), presents tape-recorded material in small steps, with expository text and question items on one channel, and commentary and review items on the other channel (Cobb, 1976, 1977).

In order to optimize the effectiveness of these tape recordings, a new approach to the measurement of study performance has been adopted. Traditional analyses of instructional effectiveness rely upon tests of comprehension or retention. Improvement in instructional materials requires hit-or-miss rewrites followed by more tests of comprehension or retention to determine whether improvement has occurred.

A much more satisfactory approach to the measurement of the differential effectiveness of instructional materials involves analyzing the learning process as it is actually taking place. The type of behaviors that students display at every stage of learning can be analyzed and used as input into the process of improving the instructional materials they study. With tape-recorded presentations, many new questions about student performance can be answered. For example: "How much time do students spend relistening to a specific part of a tape?" "Which parts of a tape do students skip over?" "Is the number of times a student relistens to one part of a tape correlated with the length of time spent listening to another part?" These and many related questions must be answered fully if we are to be able to develop truly optimized instructional materials.

Thus, a necessary and innovative tool for implementing the major goal of the Learning Through Listening project is to study how students study. This portion of our research is called the "study of study" (SOS). To implement this goal, a new minicomputerbased system has been designed and installed. The description of this system is the major thrust of this paper.

The process of human learning is difficult to study. It is easy to study how well material is learned or how fast it is learned, but measurement of the learning process while it is occurring has received relatively little attention due to the difficulties in continually monitoring what students do as they learn. For example, it is clear that a great deal of learning occurs during independent study but, to date, there is almost no objective information about what students do when they are engaged in study, specifically, how students interact with details of what is presented for learning.

In order to study the process of learning, we must continually ask a student: "What are you doing now?" Unfortunately, continually asking questions while a student is studying distracts and interferes with the learning process, making the resulting data meaningless. Therefore, to date, there has been relatively little progress in defining exactly how students learn. Relatively little data exist about what constitute effective leaming behaviors or, for that matter, optimally effective instructional materials.

An exception, of course, has been the development of some computer-assisted instructional (CAI) systems 
that record, store, and analyze student responses. But these responses are rigidly controlled by the presentation structure. Although CAI systems that allow branching and error correction have been developed, few systems allow a student to jump back, at any time, to some earlier block of material or, for that matter, to jump ahead without penalty when the material is easy, boring, or familiar. CAI emphasizes the importance of the logical flow of information in small steps and attempts by careful planning to optimize the sequential logical presentation of information, but it also constrains the study behavior of a student.

The PSI removes some of these constraints and allows somewhat more freedom with respect to the student's studying behavior (Sherman, 1974). Therefore, to avoid the pitfalls of classical noninteractive learning and still permit some freedom in studying behavior, the Learning Through Listening project chose to adapt the "talking books" traditionally supplied to the visually handicapped to the PSI format and then to closely, automatically, and unobtrusively monitor everything a student does while studying them.

This paper describes a system that unobtrusively records the responses students make while listening to tape-recorded instructional materials. In addition, the system records the exact position on the tape where all student-initiated operations occur, their durations, listening speed, time, date, and so on. All this information is placed into a readily accessible portion of a minicomputer's mass storage disk. At any time, the minicomputer can print out any desired information about the students. Since each student's data is actually sent directly to the minicomputer (this is referred to as being on-line), and the minicomputer is capable of performing calculations on the data as it comes in (referred to as real-time). The system permits on-line real-time analysis of learning through listening.

\section{FORMAT OF INSTRUCTIONAL MATERIALS}

In the Learning Through Listening project, students are given courses in a variety of subjects. The material that would normally be contained in a course in, for instance, ecology, is divided into units, which are complete subdivisions of a course. The units are divided into further subdivisions called listening segments.

Each listening segment consists of four parts: (1) The first part is called exposition. In the exposition part of the listening segment, the material is presented in a logical narrative form. (2) The second part is called commentary. In the commentary, fine points and highlights of the material are examined and presented in greater detail. (3) The third part of a listening segment is the review. It provides listening objectives for the main points made in both the exposition and commentary. (4) The fourth part is a set of questions.
The questions assess how well the student has mastered the material.

The listening segments are presented to students on standard audio tape cassettes. The exposition is presented on Channel $\mathrm{A}$ of the cassette and is followed by a short gap, an index tone, and then the questions. On the second channel of the tape, Channel B, the commentary begins at the same point as the beginning of the exposition, and after another gap at the end of the commentary, the review is presented. When a student has reached the end of a tape (one unit), the cassette is simply turned over and the remaining audio Channels A and B are listened to.

Figure 1 shows how this format is laid out on a cassette. Note that Channel $A$ is recorded on the top track of the four-track cassette and Channel B appears on Track 3. Channels A and B of the other side of the cassette are recorded on Tracks 4 and 2, respectively. This conforms to the new Library of Congress standard format for four-track cassettes.

Using standard cassette tape playback mechanisms, students are free to listen to either Channel A or B at will. They can stop, start, fast forward, or rewind the tape at any time and can even vary the speed at which the tape plays and compensate for the changed pitch of the speech, as the speed is varied through an electronic pitch-restoring network. Thus, students have considerable flexibility in how they study.

\section{THE AURAL LEARNING LABORATORY}

The general layout of the learning laboratory is presented in Figure 2. The laboratory is divided into five student cubicles and the control room. Each student cubicle contains a student tape playback machine with interface to minicomputer, speech pitch compensator, earphone adapter, and interface to intercom. It also contains a student response recorder.

\section{Student Tape Playback Machine}

The student tape playback machine is basically an American Printing House Modified Model 5192 cassette tape recorder manufactured especially for the visually handicapped to use for general-purpose recording and playback and to reproduce "talking books."

The machine is capable of playing standard cassette tapes at either $1-7 / 8$ or $15 / 16$ in./sec. It is also capable of varying playback speed by up to $100 \%$ greater or $20 \%$ less than each of the two standard fixed speeds. A student may listen either to Channel A (e.g., Track 1) of a tape or to Channel B (e.g., Track 3) of a tape. All operating controls are supplied with raised tactile symbols that inform the visually handicapped of their function. Controls include stop, play, fast forward, rewind, speed, and track select. The record and index controls have been made inoperative to prevent accidental erasure of the instructional tapes. 


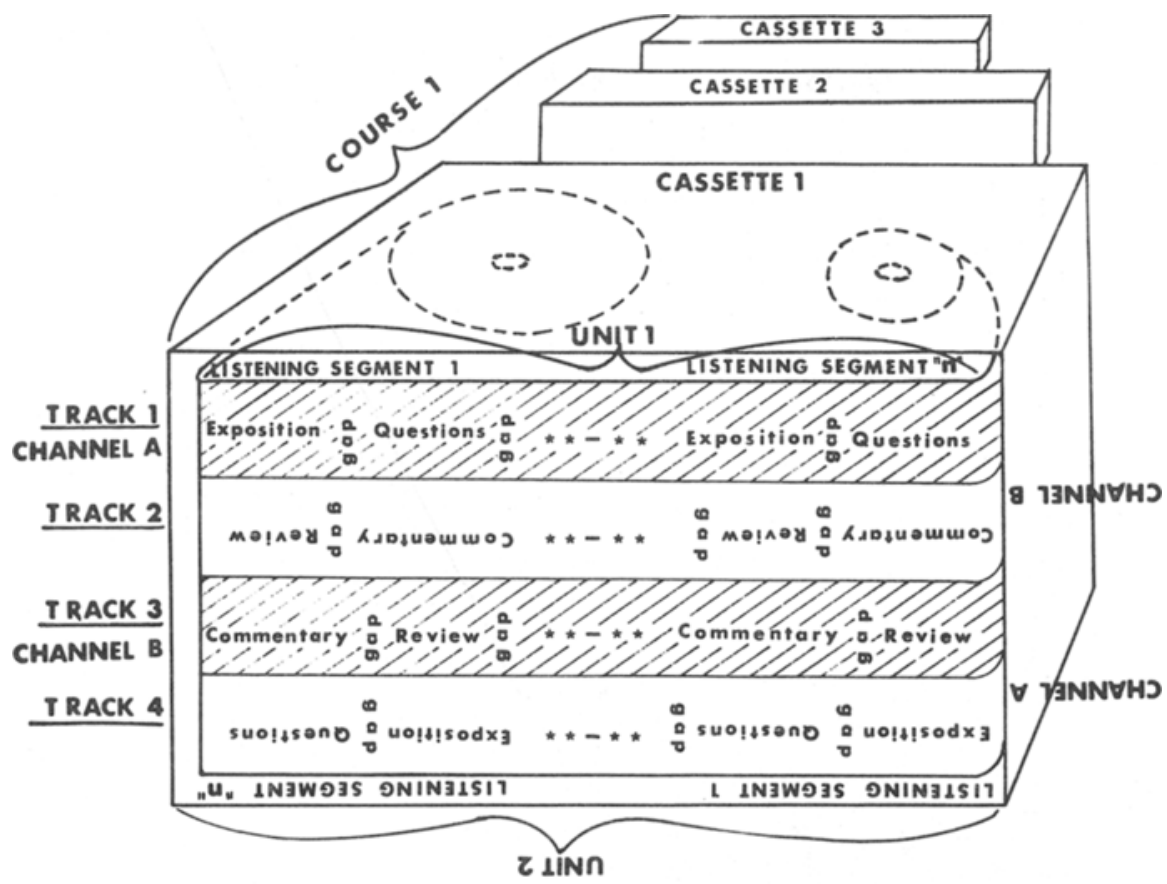

LEARNING THROUGH LISTENING FORMAT

Figure 1. The format of the tape-recorded PSI instructional materials used by the Learning Through Listening project.

Interface to computer. The modifications that have been made to the playback machines allow the minicomputer and/or another recorder to monitor every operation of the mechanism. Normally open switch contacts have been placed under the stop, play, fast forward, and rewind controls. Whenever these controls are depressed, the switch contacts close and send an information pulse to the minicomputer. An additional set of contacts on the track select switch reports to the minicomputer whenever the student changes tracks to listen to the other audio channel. A sixth switch, the tape termination button, is specially mounted on top of the machine and is used to report whenever a student stops the study of a tape.

The final modification is the most complex. The take-up reel must generate a pulse every time it rotates through one full revolution. This allows the computer to keep track of precisely where on a given tape the student is studying at any time. Although the pulses given off by the rotating take-up spindle are not linear with respect to tape footage, the pulses are consistently related to specific locations on the tape. The minicomputer simply counts the number of pulses from the start of the tape (subtracting from this count when rewinds occur) and converts these counts into centimeters using a simple software algorithm. Since the location of every part of the instructional material on the tape is known, it is a simple matter to tell precisely what material each student is studying at any time.
To accomplish this modification of the playback mechanism, it was first completely disassembled and a metal tab was attached to the take-up shaft. The tab rotates with the shaft. The main metal casting was then machined to accept a solid-state optointerrupter that has a beam of light interrupted every time the metal tab goes around. The optointerrupter consists of a light-emitting diode light source, and a photoDarlington detector element mounted in a rigid plastic case. The light-emitting diode is powered by a regulated $12 . \mathrm{V}$ dc supply that also supplies collector voltage to the photo-Darlington through a 15-kohm resistor. The output of this unit feeds directly into the input of a 9601 one-shot integrated circuit that has been adjusted to give a constant 20-msec TTL output regardless of input waveform. The 9601 is powered by its own integrated-circuit voltage regulator which drops the $12 \mathrm{~V}$ to the required $5 \mathrm{~V}$. The $12 \mathrm{~V}$ is supplied externally by the minicomputer.

The seven data outputs from each student playback mechanism are stop, play, fast forward, rewind, track select, tape termination, and tape position count. A 20-conductor-ribbon cable carries this data from a connector mounted on the playback mechanism through a wall to the minicomputer. Alternate wires in the cable are wired as ground lines to provide protection from cross-talk and noise.

Speech compensator. A variable speech compensator is plugged directly into the output of the playback mechanism. The speech compensator allows a student 


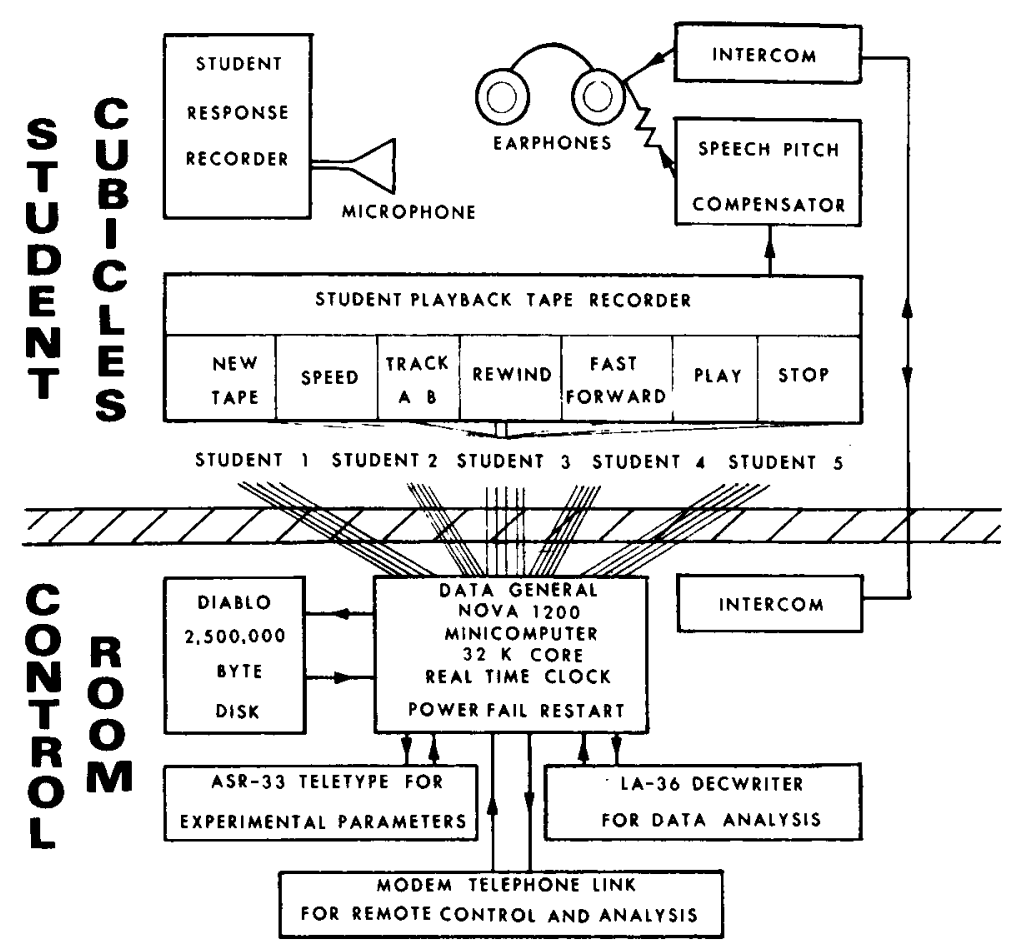

Figure 2. Block diagram of the student cubicles and control room of the Learning Through Listening project.

to correct the pitch of the voice on a tape that is being played at a higher or lower than normal speed. For instance, a student listening to difficult material may wish to slow down the vocal presentation. This is done by first adjusting the tape speed control on the playback mechanism for a slower speed and then adjusting the tape speech compensator until the speech pitch sounds normal. A dual-ganged potentiometer will eventually be installed to allow single-knob control of both tape speed and pitch.

Earphone adapter. The output of the speech compensator is a low-impedance (about 4-ohm) signal that can easily drive a set of earphones. However, it is also necessary to have a medium-impedance intercom and the earphone output of another Model 5192 tape recorder mix together so that they all drive the earphones at approximately the same level. To achieve this, a 150 -ohm resistor is placed in series with the output of the speech compensator. This forms one leg of a three-way connection that terminates in a jack into which a standard pair of earphones is plugged.

Interface to intercom. Since it is important to be able to communicate easily and directly with each student, an intercom was installed in front of each student and connected to their earphones. A 47-microfarad dc blocking capacitor preserves the "call" feature of the intercom. It has been found satisfactory to simply connect the intercom to the student earphone through this blocking capacitor. A light-emitting diode, driven directly by the high-impedance winding of the intercom output transformer, installed in each master intercom in the control room, gives a visual indication and an auditory call signal whenever a student requests assistance. An earphone jack allows more intelligible conversations with the students.

\section{Student Response Recorder}

Every student has a second American Printing House 5192 tape recorder that is used for recording verbal responses to questions. The recorder is not modified in any way, but the earphone jack is connected directly to the student's earphones. A separate microphone with an on-off switch is supplied so that the student can easily control the operation of the tape recorder.

\section{BACK-UP RECORDING SYSTEM}

Since development of the necessary hardware and software to fully utilize the capabilities of the minicomputer was going to take months, a 40-channel paper-chart recorder was installed temporarily. The recorder served to record data using 35 ink pens. Whenever a student pressed any key on the playback machine, the input of a 75451 integrated circuit $(40 \mathrm{~mA})$ peripheral driver was grounded. This caused the output to switch $24 \mathrm{~V}$ to ground and activate one of the pen coils. This in turn wrote a small blip on the constantly moving chart paper. All information could then be read directly off the chart paper with the exception of the speed of the tape recorder, which was calculated by measuring the distance between the tape position counter pulses. Because of the huge 
volume of paper-chart data generated by this recorder, it was abandoned as soon as the minicomputer was installed.

In addition to the chart recorder, it is desirable to be able to obtain immediate counts of how much tape is being listened to while playing the tape forward and in reverse. Ten tiny pocket calculators were hooked up to the tape position counter pulses and, through AND gates, to play, fast forward, or rewind pulse outputs, These calculators were modified to act as counters by connecting a switching transistor's emitter and collector directly across their ADD-key contacts. When the appropriate pulses were received by the AND gate, it turned on the switching transistor connected directly across the ADD key of the calculator and the calculator incremented by one. The counters can be connected to any of the playback machine functions and give instantaneous information about the number of times that a given function has been activated.

The graphic recorder and the counters were plugged into the middle of the 20-conductor-ribbon cable coming from each student playback machine. They can be connected or disconnected at any time without interfering with the minicomputer. This provides a totally independent back-up system that functions with or without the minicomputer and assures that no data are lost.

\section{MINICOMPUTER HARDWARE}

The minicomputer is a Data General Corporation Nova 1200 central processor with 32,000-word core memory, real-time clock, power-fail protection, and Teletype interfaces modified for selectable baud rates. It is interfaced to a 2.5 -million-word capacity Diablo 4047A disk drive by a Diablo 4046 interface and 4047 adapter. A Teletype Corporation ASR-33 teleprinter provides printed data output and communication between the operator and the data-acquisition program. An LA-36 Decwriter provides data analysis at 300 baud. A modem allows data to be accessed from diverse remote locations over the telephone lines.

A specially constructed interface designed by Coldspring Instrument Corporation (Chicken Valley Road, Locust Valley, New York 11560) receives contact closures or pulses from each of the student playback mechanisms and converts them to TTL pulses that are acceptable to the minicomputer. Each of the 20 conductor cables coming from the student playback machines plug into the interface at the back of the central processing unit.

The hardware allows data to enter the central processing unit via the specially constructed interface, to be formatted in core memory, and then recorded on the mass storage disk. The disk, with its capacity of 2.5 million words, can record approximately 56 days of data. When the disk is full, the disk cartridge is simply removed and a fresh disk cartridge is inserted in the disk drive. The full disk cartridges are taken to Coldspring Instrument Corporation and the data is recorded on magnetic tape as a safety precaution in case the original disk is accidentally lost. Control of these pieces of computer hardware as well as data analysis is the function of comprehensive FORTRAN IV-based software developed for Learning Through Listening by Coldspring Instrument Corporation. This software is described in the next section of this report.

\section{MINICOMPUTER SOFTWARE}

In order to save time and software development costs, the software was developed around the Data General Corporation real-time disk operating system (RTDOS) software package. This system supports a versatile real-time FORTRAN IV language and is capable of foreground-background programming. In this mode, the minicomputer is continually accepting data from the student playback machines and placing this data into the appropriate locations in memory. At the same time, during the inevitable gaps and pauses in the dataacquisition program, another program of printing out the data or calculating correlations between segments of previously recorded data is being performed.

The RTDOS, as most similar systems, has a command mode during which the computer operator can issue commands to the software by simply typing them on the teletypewriter. This mode is called the command line interpreter (CLI). The available commands permit a wide variety of chores to be performed such as loading programs, linking, relocating, and running programs. Using the RTDOS, Coldspring Instrument Corporation has written a program in real-time FORTRAN IV, which performs all of the required data-acquisition and storage functions as well as having the capability of linking up with a powerful statistical subroutine package for the performance of virtually any kind of statistical analysis. Figure 3 is a block diagram of the data analysis software which works as follows.

\section{Initialization}

At the beginning of each day, the minicomputer is started and the current time and date are entered. Any housekeeping chores such as consolidating files or listing file names are then performed. The executive program is then loaded; this initializes the Teletype terminal. The experimental parameters are entered, including the student cubicle number, the number of the student using that machine, the number of the tape being used in that machine, and the name of the disk file into which that student's data are to be entered. This is done for each of the machines that will be in use. The Teletype terminal then prints out every response, and all associated data or the printout may 


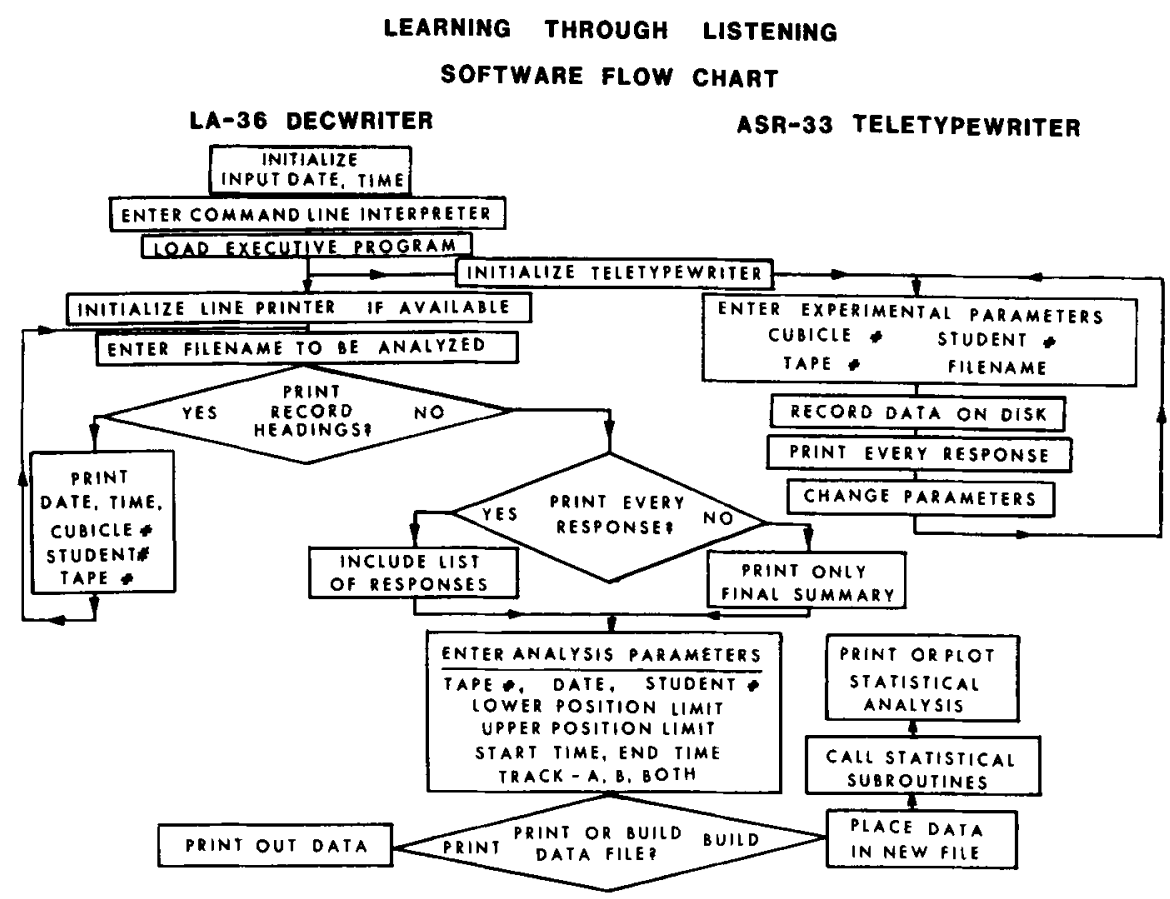

Figure 3. Block diagram of the data-acquisition and data-analysis software.

then be inhibited so that the data is simply entered on the disk.

Whenever a student presses the tape termination switch, data acquisition from that particular student playback machine and cubicle is immediately terminated. When a student wishes to listen to a new tape on the machine, the machine number, student number, tape number, and file name are reentered.

\section{On-Line, Real-Time Data Acquisition}

The minicomputer software maintains a continuously running, real-time clock that keeps track of the exact time and date after it is initialized. All events that occur during a daily session are recorded and stored on the mass storage disk along with information from the minicomputer's real-time clock, which tells precisely at what time and date any event occurred.

Whenever a student makes any kind of response or change in the operation of the student playback machine, it is immediately recorded in combination with information from the real-time clock. In order to completely specify each event of this type, a considerable amount of information must be recorded at each occurrence. This information is necessary in order to assure that all of the data can be accessed and retrieved. For one purpose it may be desirable to retrieve the data by student number; for a different type of analysis it may be desirable to retrieve data by tape number or by day. Therefore, all information that can possibly be needed must be recorded along with each student-initiated event.

For example, say that Student 33 is listening to Tape 27 on Channel B at a speed of $6 \mathrm{~cm} / \mathrm{sec}$ on student playback Machine 5. If the student presses the STOP key at a location 312 counts from the beginning of the tape, the minicomputer records all of the following information on the mass storage disk: Machine 5, Student 33, Tape 27, date, exact time, STOP, Channel B, tape counter Location 312.

With all of this information recorded for every event, data may be readily retrieved by any of the following specifiers: machine number, student number, tape number, date, time, which of the two available tracks are being listened to, and location on the tape. Since all of the information is stored on the disk, an almost unlimited number of different types of analysis may be performed on the data.

\section{Data Analysis of Software}

Data analysis may be performed either during the time that the subjects are learning or at any later time.

The types of data analysis that can be performed range from the simplest sequential repetition of the time course of events to the most complex forms of statistical analysis. To save time and software development cost, relevant portions of the comprehensive scientific subroutine package (SSP) are included on each mass storage disk. The package consists of over 300 scientific subroutines for the analysis and graphing of data. Any subroutine can be selected and used to provide statistical analysis of any desired data stored on the mass storage disk.

For instance, suppose we desire to obtain the arithmetic mean and variance of the amount of time that Student 33 spent listening to the "commentary" 
part of all listening segments. The operator can readily retrieve a list of these times from the mass storage disk by specifying the desired information as the analysis program is entered. Then, the mean and variance subroutine may be called up from the SSP and used to quickly perform the desired calculations. If a frequency distribution is desired, another subroutine will plot it out on the Decwriter in a graphical form that is suitable for publication.

Data is accessed by precisely specifying all the desired data parameters either on the Decwriter or from a remote terminal via the modem.

The following information must be provided in order to access data: file name, tape number, date (month, day, year), student number, tape position count for lower limit of analysis, tape position count for upper limit of analysis, time to begin analysis, time to end analysis, and Track A or B, or both.

Data may be printed out or placed in a file for statistical analysis. If it is placed in a file, a call to the statistical analysis subroutine package will cause a statistical analysis to be performed and the results to be typed or plotted on the terminal.

\section{Some Preliminary Results}

Visually impaired adults, recruited from the greater New York City area, are paid on an hourly basis for their participation in the Learning Through Listening project. Their motivation is high not only because they are being paid, but also because they know that their performance is being monitored and because of the contingencies of the PSI.

Since November 1976, the Learning Through Listening project has been obtaining study activity data and comprehension scores from the student subjects. Each subject masters each unit of course material by independently studying each listening segment, receiving immediate feedback on the correctness of responses and from individual tutoring following each taperecorded unit-evaluation test. Approximately $80 \%-90 \%$ of all learning time is spent independently of tutorial contact. All subjects, most of whom have completed some high school education, report that the dual-track aural adaptation structure is a large improvement over the aural study of literally transcribed textbooks.

As the students progress through the initial listening segments of the first course, they learn to operate the playback mechanisms, the intercom, and the student response tape recorder. Their learning behavior is initially variable but, after a few weeks, consistent patterns of study behavior begin to emerge. Average study times decrease as they discover more efficient study patterns. Although their scores on questions remain consistently high, the study times needed to achieve these scores decrease.

Study activities are distributed differently across the four parts of a listening segment. In terms of both frequency and duration of all playback operations, the amount of study activity is lowest for the exposition, followed by the commentary and review, and highest for the question part. The computer printout and analysis of the exact positions that students rewind to most frequently provide information about which portions of the listening segment have not been assimilated and need revision.

In addition to the direct measures of study activity provided by the computer, a number of new measures may be derived from the recorded data that provide useful information about study activity. For example, by dividing the total amount of active study time by the total duration of the tape recording, we obtain a ratio that reflects the relative amount of time needed to study a recorded presentation of a given length. And by dividing that ratio (the study-time ratio) by the average comprehension scores, we obtain a new ratio (the difficulty ratio), which is an index of the relative difficulty of the presentation.

The minicomputer provides access to a great deal of information about what students do as they study instructional material. This data is being used both to optimize the instructional material and to study how students study in a relatively free learning environment. Future plans for the Learning Through Listening project include detailed analysis of all data and the concomitant recording of electromyographic subvocalization patterns in an attempt to obtain a full understanding of the behavior of learning.

\section{REFERENCES}

Совв, E. S. Learning through listening: A New approach. Journal of Visual Impairment and Blindness, 1976, 77, 302-308.

CoBB, E. S. Learning through listening: Final report. (JanuaryJune 1976 and July-June 1977). New York State Department of Education, Bureau of Special Continuing Education.

Sherman, J. Y. Personalized system of instruction: 41 germinal papers. Menlo Park, Calif: W. A. Benjamin, 1974. 